\title{
Late-Evening Carbohydrate and Branched-Chain Amino Acid Snacks Improve the Nutritional Status of Patients Undergoing Hepatectomy Based on Bioelectrical Impedance Analysis of Body Composition
}

\author{
Kosuke Kobayashia Junichi Kaneko ${ }^{a}$ Takamune Yamaguchi ${ }^{a}$ \\ Yoshikuni Kawaguchi $^{a}$ Junichi Arita ${ }^{a}$ Nobuhisa Akamatsu ${ }^{a}$ \\ Takeaki Ishizawa $^{a}$ Rie Sekine ${ }^{b}$ Hideaki ljichi $^{b}$ Naoto Kubotab \\ Kazuhiko Fukatsu ${ }^{c}$ Norihiro Kokudod Kiyoshi Hasegawa ${ }^{a}$

\begin{abstract}
a Hepato-Biliary-Pancreatic Surgery Division and Artificial Organ and Transplantation Division, Department of Surgery, Graduate School of Medicine, The University of Tokyo, Tokyo, Japan; ${ }^{b}$ Department of Clinical Nutrition Therapy, The University of Tokyo, Tokyo, Japan; ' Surgical Center, The University of Tokyo, Tokyo, Japan; dNational Center for Global Health and Medicine, Tokyo, Japan
\end{abstract}

\section{Keywords}

Late-evening snack - Nutritional status - Hepatectomy - Bioelectrical impedance analysis . Hepatocellular carcinoma $\cdot$ Colorectal liver metastases

\begin{abstract}
Background: This prospective study measured body composition based on bioelectrical impedance analysis (BIA) in relation to preoperative and postoperative nutritional support and status in patients undergoing liver surgery. Methods: Thirty-seven patients with impaired liver function (indocyanine green retention rate at $15 \mathrm{~min}>15 \%$ ) undergoing hepatectomy for hepatocellular carcinoma or colorectal liver metastasis were enrolled. The control group $(n=$ 10) received no nutritional supplementation. The late-evening snack (LES, $n=26$ ) group received a 210-kcal snack comprising a carbohydrate with branched-chain amino acids for 2 weeks before surgery through to 12 weeks after surgery. BIA of body composition, including body cell mass and skeletal muscle volume, was performed. Results: Although there was no sarcopenia based on the consensus report of the Asian Working Group 2 weeks before surgery, the skeletal muscle volumes in the control and LES groups were at the lower limit of the normal range. Body cell mass and skeletal muscle volume were significantly lower in the control group than in the LES group at $4(p=0.03)$ and $12(p=0.02)$ weeks after surgery. Conclusion: Late-evening carbohydrate and branched-chain amino acid snack supplementation may improve nutritional status in patients with impaired liver function undergoing hepatectomy.
\end{abstract}




\section{Introduction}

Hepatectomy is a curative treatment option for hepatocellular carcinoma [1] and colorectal liver metastasis [2]. Liver surgery is a challenging procedure for patients. Major morbidity in malignant liver disease reaches $27 \%$ [3]. Nutritional support is one of several multimodal pathways to enhance recovery after surgery [4]. The current Enhanced Recovery after Surgery (ERAS) guidelines for liver surgery recommend that only malnourished patients receive oral nutritional supplements [4]. In a non-surgical setting, however, cirrhotic patients are already malnourished, and the European Society for Clinical Nutrition and Metabolism (ESPEN) guidelines recommend late-evening carbohydrate snacks to improve their nutritional status [5-7]. No surgical guidelines for perioperative care, however, recommend routine nutritional support for cirrhotic patients and/or patients with abnormal liver function $[4,8]$.

Over the last 2 decades, several trials have been performed to evaluate the value of nutritional support during the perioperative period of hepatectomy [9-12]. The results of many of these studies suggest that regular nutritional support should be part of a surgical protocol, but the findings are inconsistent [13]. No consensus for a nutritional support protocol is reported for hepatectomy patients. In addition to the effect of nutritional support on clinical outcome, it is crucial to precisely evaluate the patient's nutritional status, but reports of surgical patients' nutritional status during the perioperative period are scarce. The ESPEN guidelines recommend determining the precise nutritional status based on measurement of body composition by bioelectrical impedance analysis (BIA) [8]. Body composition shifts occur before physiologic changes and provide useful and precise information for evaluating nutritional status [14]. BIA allows for non-invasive, low-cost monitoring of the body composition in humans based on the conduction of a low-intensity alternating electric current [15]. Body cell mass (BCM), a body composition parameter comprising metabolically active tissue or cells in the body that can eliminate extracellular water (ECW), reliably indicates edema due to surgical stress [16]. ESPEN guidelines recommend measuring BCM as an indicator of body composition for evaluating the nutritional state in patients with liver disease $[17,18]$. To date, there are no reports of BIA of body composition measurements in relation to the perioperative nutritional status in patients undergoing hepatectomy. The present prospective, comparative, feasibility study investigated whether late-evening carbohydrate and branchedchain amino acid (BCAA) snacks affect BCM based on BIA and other aspects of the nutritional status of patients with impaired liver function undergoing hepatectomy for hepatocellular carcinoma and colorectal liver metastasis.

\section{Materials and Methods}

Study Design

The present study was a non-randomized, prospective trial to explore the feasibility of nutritional support under BIA for monitoring. The scheduled allocation during a 5-year study period was that the initial 10 consecutive patients would be assigned to the control group and the next 30 consecutive patients would be assigned to the late-evening snack (LES) group and received nutritional support. The number of patients was based on the frequency of impaired liver function patients with an indocyanine green (ICG) retention rate at 15 min greater than $20 \%$, and represents one-fifth of the patients in our previous report [19].

The inclusion criteria were patients over 18 years of age with impaired liver function, ICG retention rate at 15 min greater than 15\%, scheduled to undergo liver resection for hepatocellular carcinoma or colorectal liver metastasis. The exclusion criteria were urgent operation, extrahepatic bile duct resection, impaired renal function (serum creatinine level $>1.5 \mathrm{mg} / \mathrm{dL}$ ), prior nutritional support, and a history of anaphylaxis to ICG or evident allergy to iodine. All enrolled subjects provided written informed consent. 
The surgical indications for hepatocellular carcinoma and colorectal liver metastasis were described in previous reports $[20,21]$. The hepatectomy procedure was based on our institutional policy according to the tumor extent [22]. A major hepatectomy was defined as resection of four or more Couinaud segments and a minor hepatectomy was defined as the resection of no more than three segments [23]. Surgical site infection was defined according to the criteria of the National Nosocomial Infections Surveillance System [24]. The protocol of postoperative physical therapy is that the patient starts sitting at the bed side and walking in the hospital ward from the day after surgery. A nurse and physical therapist help the patient with their own task lists, increasing walking time and distance, under the supervision of rehabilitation doctors. The physical therapist checks the status of all patients according to the same protocol every month for 3 months after discharge.

\section{Late-Evening Carbohydrate with BCAA Snack Supplementation}

The nutritionist provided individual nutritional counseling for each patient 2 weeks before and every 4 weeks after surgery up to 12 weeks. All patients were supplied a meal comprising $35-40 \mathrm{kcal} / \mathrm{kg}$ daily. The initial 10 patients were assigned to the control group and received no supplementation. Subsequent patients were assigned to the LES group and were provided a 210-kcal snack comprising a carbohydrate with BCAA (Aminoleban EN powder mix; Otsuka Pharmaceutical Co. Ltd., Tokyo, Japan) every night at 10 p.m. for 2 weeks before surgery through to 12 weeks after surgery.

\section{Evaluation of Preoperative and Postoperative Variables and Nutritional Status}

Two weeks before surgery, the Child-Pugh score, platelet count, serum total bilirubin level, serum creatinine level, and estimated glomerular filtration rate were measured, and the presence of sarcopenia was evaluated in all patients based on the consensus report of the Asian Working Group for Sarcopenia [25]. A nutritionist evaluated the nutritional status based on a subjective global assessment [26].

At 2 weeks before surgery, and at 1, 4, and 12 weeks after surgery, hematologic and biochemical tests were carried out, including total lymphocyte count (normal range 1,300-4,000/ $\mu \mathrm{L}$ ), serum levels of albumin (4.1-5.1 g/dL), pre-albumin (19.5-38.7 mg/dL), total cholesterol (142-248 mg/dL), cholinesterase (240-486 $\mathrm{U} / \mathrm{L}$ ), transferrin (men 190-300, women 200-340 mg/dL), and retinol binding protein (men 2.7-6.0, women 1.9-4.6 mg/dL). Postoperative morbidity and mortality were evaluated according to the Clavien-Dindo classification [27]. Re-hospitalization was defined as re-admission within 30 days after the first discharge from the hospital.

\section{BIA of Body Composition}

At 2 weeks before, and at 1, 4, and 12 weeks after surgery, body mass index, body weight, BCM (normal range 29.9-36.6 kg), skeletal muscle volume (25.4-31.1 kg), arm circumference (men 27.2 \pm 3.0 , women 25.3 $\pm 3.1 \mathrm{~cm}$ ), arm muscle circumference (men $23.7 \pm 2.8$, women $20.3 \pm 2.6 \mathrm{~cm}$ ), and the ratio of the ECW to total body water (ECW/TBW, 0.36-0.40) were evaluated by direct segmental multi-frequency BIA using a body composition analyzer (Inbody 720 and/or Inbody S10; InBody Japan Inc., Tokyo, Japan).

\section{Statistical Analysis}

Variables are expressed as the median (range) and compared between groups using Fisher's exact test, the $\chi^{2}$ test where appropriate, and Wilcoxon's rank-sum test. The change rate of variables between groups was analyzed by repeated measures analysis of variance using JMP software (version 12.2.0; SAS Institute Inc., Cary, NC, USA). A $p$ value $<0.05$ was considered to indicate statistical significance.

\section{Results}

A total of 345 patients were screened for enrollment between August 2012 and the end of December 2016. Among those, the ICG retention rate at 15 min was less than $15 \%$ in 280 patients. The remaining 65 patients had an ICG retention rate greater than $15 \%$ at 15 min and were diagnosed with impaired liver function. None of the patients were ICG intolerant. Twenty-eight patients were excluded from the analysis as follows: 19 required an urgent operation, 5 had impaired renal function, and 4 had received prior nutritional support. A total 


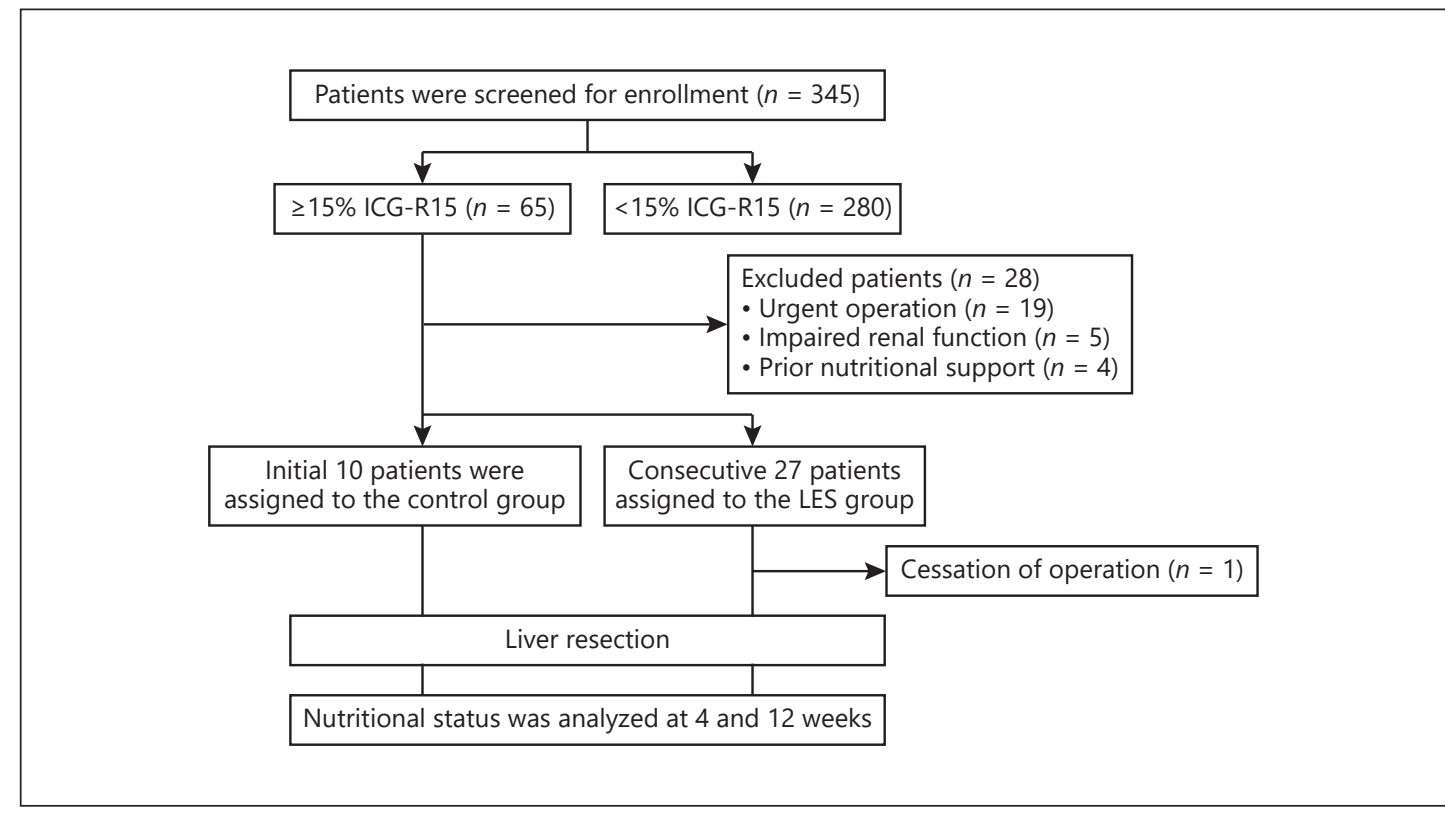

Fig. 1. Flowchart of the study participants. ICG, indocyanine green; LES, late-evening snack.

of 37 patients were enrolled and the initial 10 patients were assigned to the control group. Twenty-seven consecutive patients were assigned to the LES group. In the LES group, 1 patient for whom the operation was canceled was excluded from the present analysis. Finally, a total of 36 patients were prospectively evaluated (Fig. 1).

\section{Patient Characteristics}

Patient characteristics were comparable between groups (Table 1). The indications for hepatectomy included hepatocellular carcinoma (control, $n=8$ vs. LES, $n=22, p=0.99$ ) and colorectal liver metastasis (control, $n=2$ vs. LES, $n=4, p=1.00$ ). The platelet count was significantly lower in the control group than in the LES group $(p=0.02)$. The values of the other variables were not significantly different.

\section{Surgical Outcomes}

The rate of major hepatectomy, estimated blood loss, amount of blood transfused, Clavien-Dindo classification, and hospital stay were similar between the groups (Table 2). The operation time tended to be shorter in the control group than in the LES group ( $p=0.07)$, and the rate of surgical site infection tended to be lower in the LES group than in the control group ( $p=0.10)$. There was no significant difference in the intraoperative fluid balance ( $p=$ $0.72)$, the length of the intensive care unit stay $(p=0.74)$, and the length of oral intakes after hepatectomy $(p=0.88)$. The first day of defecation was significant earlier in the LES group ( 3 days, range 2-7) than in the control group (5 days, range $2-7 ; p=0.01$ ). A patient in the LES group died of pneumonia 43 days after right hemi-hepatectomy for hepatocellular carcinoma classified as Clavien-Dindo grade V. The overall 90-day mortality rate was $2.7 \%$.

\section{Nutritional Outcomes}

Nutritional status, including the outcome of the body composition analysis, is summarized in Table 3. None of the patients had sarcopenia, but the skeletal muscle volume in the control (24.0 kg) and LES (25.4 kg) groups was at the lower limit of the normal range (25.4- 
Table 1. Patient characteristics

\begin{tabular}{lccc}
\hline & $\begin{array}{l}\text { Control group } \\
(n=10)\end{array}$ & $\begin{array}{l}\text { LES group } \\
(n=26)\end{array}$ & $p$ value \\
\hline Age, years & $74(46-77)$ & $76(48-85)$ & 0.33 \\
Sex (male/female) & $5 / 5$ & $21 / 5$ & 0.13 \\
Body mass index & $24.0(19.8-33.0)$ & $23.4(17.4-31.4)$ & 0.54 \\
Subjective global assessment A/B/C & $7 / 3 / 0$ & $22 / 4 / 0$ & 0.37 \\
Child-Pugh score & $5(5-6)$ & $5(5-7)$ & 0.41 \\
Platelet count, $\times 10^{4} / \mu \mathrm{L}$ & $10.1(7.1-36.9)$ & $15.4(11.1-32.3)$ & $0.02^{*}$ \\
Total bilirubin, mg/dL & $0.9(0.5-1.6)$ & $0.7(0.4-1.7)$ & 0.35 \\
PT-INR & $1.02(0.91-1.09)$ & $0.98(0.86-1.91)$ & 0.33 \\
Serum creatinine, mg/dL & $0.73(0.43-1.65)$ & $0.88(0.52-1.71)$ & 0.05 \\
eGFR, mL/min/1.73 m ${ }^{2}$ & $69.8(35.8-104.5)$ & $62.0(25.9-102.3)$ & 0.24 \\
HbA1c, \% & $5.3(4.3-7.1)$ & $5.6(4.2-7.1)$ & 0.38 \\
ICG-R15, \% & $19.5(15.8-45.2)$ & $17.9(15.3-33.7)$ & 0.20 \\
Disease & & & 0.31 \\
Hepatitis B or C & 6 & 6 & 0.30 \\
Non-alcoholic steatohepatitis & 4 & 22 & 1.00 \\
Hepatocellular carcinoma & 8 & 4 & 1.00 \\
Colorectal liver metastasis & 2 & 12 & 0.13 \\
Background of liver & & $14(4)$ & 0.07 \\
Cirrhosis & $2(2)$ & & \\
Chronic hepatitis (prior chemotherapy) & & & \\
\hline
\end{tabular}

Data are presented as $n$ or the median (range). ${ }^{*} p<0.05$. PT-INR, prothrombin time-international normalized ratio; eGFR, estimated glomerular filtration rate; HbA1c, Hemoglobin A1c; ICG-R15, indocyanine green retention rate at $15 \mathrm{~min}$.

Table 2. Surgical outcomes

\begin{tabular}{lccc}
\hline & $\begin{array}{l}\text { Control group } \\
(n=10)\end{array}$ & $\begin{array}{l}\text { LES group } \\
(n=26)\end{array}$ & $p$ value \\
\hline Major hepatectomy & 0 & $4(15.4)$ & 0.56 \\
Operation time, min & $272(116-553)$ & $392(204-800)$ & 0.07 \\
Estimated blood loss, mL & $365(35-3,650)$ & $454(140-5,103)$ & 0.29 \\
Transfusion & $3(30.0)$ & $7(26.9)$ & 1.00 \\
Surgical site infection & $5(50.0)$ & $5(19.2)$ & 0.10 \\
Clavien-Dindo classification I or II & $5(50.0)$ & $11(42.3)$ & 0.72 \\
Clavien-Dindo classification III or IV & 0 & $1(3.9)$ & 1.00 \\
Clavien-Dindo classification V & 0 & $1(3.9)$ & 1.00 \\
Hospital stay, days & $18(11-23)$ & $13(8-51)$ & 0.15 \\
\hline
\end{tabular}

Data are presented as $n(\%)$ or the median (range).

$31.1 \mathrm{~kg}$ ). At 2 weeks before surgery, the nutritional status did not differ significantly between the groups. At 4 weeks after surgery, BCM was significantly lower in the control group (26.3 $\mathrm{kg}$, range 13.8-37.0; normal range 29.9-36.6) than in the LES group (30.0 kg, range 20.8$37.3 ; p=0.03)$. Skeletal muscle volume was also significantly lower in the control group (21.9 $\mathrm{kg}$, range $10.6-31.7)$ than in the LES group $(25.3 \mathrm{~kg}$, range $16.9-31.9)$ at 4 weeks after surgery ( $p=0.02$ ). Other variables, including lymphocyte count, serum albumin, pre-albumin, total cholesterol, cholinesterase, transferrin, retinol binding protein, body weight, ECW/TBW, arm circumference, and arm muscle circumference did not differ significantly between the groups. 
Table 3. Nutritional outcomes

\begin{tabular}{|c|c|c|c|c|}
\hline & \multirow[t]{2}{*}{2 weeks prior } & \multicolumn{3}{|l|}{ Control group } \\
\hline & & 1 week & 4 weeks & 12 weeks \\
\hline \multicolumn{5}{|l|}{ Hematologic and biochemical tests } \\
\hline Lymphocyte count/ $\mu \mathrm{L}$ & $1,350(990-2,926)$ & $1,769(657-2,867)$ & $1,482(806-2,408)$ & $1,515(1,088-5,529)$ \\
\hline Serum albumin, g/dL & $3.5(2.6-4.1)$ & $3.0(2.7-3.7)$ & $3.1(2.9-3.5)$ & $3.5(3.0-4.1)$ \\
\hline Pre-albumin, mg/dL & $13.1(8.3-21.4)$ & $11.7(9.2-18.4)$ & $10.7(7.3-19.7)$ & $15.0(7.5-21.7)$ \\
\hline Total cholesterol, mg/dL & $156(114-286)$ & $120(90-149)$ & $119(88-178)$ & $159(141-238)$ \\
\hline Cholinesterase, U/L & $200(128-88)$ & $123(81-193)$ & $150(72-205)$ & $179(137-322)$ \\
\hline Transferrin, mg/dL & 270 (197-389) & $175(128-295)$ & $230(145-332)$ & $304(185-333)$ \\
\hline Retinol binding protein, $\mathrm{mg} / \mathrm{dL}$ & $1.7(1.0-3.3)$ & $1.9(1.1-2.9)$ & $1.6(1.1-3.3)$ & $2.0(0.8-3.1)$ \\
\hline \multicolumn{5}{|l|}{ BIA of body composition } \\
\hline Body weight, kg & $55.8(42.1-86.3)$ & $57.7(46.2-80.8)$ & $53.8(38.5-79.6)$ & $57.3(37.3-82.3)$ \\
\hline BCM, kg & $28.5(15.9-40.0)$ & $27.6(17.7-37.0)$ & $26.3(13.8-37.0)$ & $26.7(13.7-38.0)$ \\
\hline Skeletal muscle volume, kg & $24.0(12.5-34.4)$ & $23.1(14.2-31.5)$ & $21.9(10.6-31.7)$ & $22.3(10.5-32.6)$ \\
\hline Arm circumference, $\mathrm{cm}$ & $28.9(26.4-34.1)$ & $29.2(26.4-34.1)$ & $28.1(25.2-33.3)$ & $28.6(25.7-33.0)$ \\
\hline Arm muscle circumference, $\mathrm{cm}$ & $22.2(17.6-25.6)$ & $22.3(18.4-25.6)$ & $20.4(16.6-23.5)$ & $21.8(16.4-24.4)$ \\
\hline \multirow[t]{3}{*}{ ECW/TBW } & $0.389(0.386-0.404)$ & $0.401(0.386-0.425)$ & $0.390(0.381-0413)$ & $0.396(0.389-0.402)$ \\
\hline & 2 weeks prior & \multicolumn{3}{|l|}{ LES group } \\
\hline & & 1 week & 4 weeks & 12 weeks \\
\hline \multicolumn{5}{|l|}{ Hematologic and biochemical tests } \\
\hline Lymphocyte count/ $\mu \mathrm{L}$ & $1,682(792-4,300)$ & $1,800(802-9,000)$ & $1,600(700-3,100)$ & $1,900(800-3,360)$ \\
\hline Serum albumin, g/dL & $3.8(2.1-4.5)$ & $3.3(2.4-3.9)$ & $3.5(2.7-4.1)$ & $3.7(2.9-4.3)$ \\
\hline Pre-albumin, mg/dL & $17.5(9.3-29.0)$ & $12.0(8.9-17.2)$ & $14.4(5.9-22.7)$ & 14.5 (10.4-23.1) \\
\hline Total cholesterol, mg/dL & $167(80-301)$ & $127(78-158)$ & $147(79-215)$ & 167 (124-199) \\
\hline Cholinesterase, U/L & $239(109-347)$ & 147 (67-259) & $189(39-286)$ & $222(113-338)$ \\
\hline Transferrin, mg/dL & $242(143-364)$ & $182(126-260)$ & $241(163-320)$ & $275(173-379)$ \\
\hline Retinol binding protein, $\mathrm{mg} / \mathrm{dL}$ & $2.1(1.4-3.4)$ & $1.7(0.7-2.9)$ & $1.9(1.1-3.0)$ & $2.1(1.3-3.2)$ \\
\hline \multicolumn{5}{|l|}{ BIA of body composition } \\
\hline Body weight, kg & $63.1(45.8-95.6)$ & $64.9(46.7-91.7)$ & $62.6(47.4-92.8)$ & $62.2(48.3-94.9)$ \\
\hline $\mathrm{BCM}, \mathrm{kg}$ & $30.1(20.2-39.0)$ & $30.7(22.8-37.1)$ & $30.0(20.8-37.3)^{*}$ & $25.2(20.2-31.7)$ \\
\hline Skeletal muscle volume, $\mathrm{kg}$ & $25.4(18.1-33.5)$ & $26.2(18.7-31.8)$ & $25.3(16.9-31.9)^{*}$ & $25.2(20.2-31.7)$ \\
\hline Arm circumference, $\mathrm{cm}$ & $30.0(23.7-37.1)$ & $30.1(24.0-37.2)$ & $29.1(23.5-36.6)$ & $28.5(24.5-37.5)$ \\
\hline Arm muscle circumference, $\mathrm{cm}$ & $22.1(19.6-27.7)$ & $22.9(18.4-26.2)$ & $22.1(18.2-27.0)$ & $21.7(18.8-26.4)$ \\
\hline ECW/TBW & $0.391(0.380-0.407)$ & $0.400(0.375-0.430)$ & $0.393(0.375-0.408)$ & $0.393(0.375-0.409)$ \\
\hline
\end{tabular}

Data are presented as the median (range). ${ }^{*} p<0.05$ compared to the controls. BCM, body cell mass; LES, late-evening snack; ECW/ TBW, extracellular water/total body water ratio.

When the variables of nutritional status were defined as $100 \%$ at 2 weeks before hepatectomy, the rate of change of BCM in the control and LES groups was 94.0 versus $98.0 \%$ at 4 weeks ( $p=0.03$, Fig. $2 \mathrm{a}$ ); body weight at 4 and 12 weeks was 93.2 versus $97.6 \%(p<0.01)$ and 96.2 versus $99.1 \%(p<0.01$, Fig. $2 \mathrm{~b})$; skeletal muscle volume at 4 weeks was 93.9 versus $97.5 \%$ ( $p=0.02$, Fig. 2c); transferrin at 4 weeks was 79.0 versus $97.5 \%(p<0.01$, Fig. 2 d) , and arm muscle circumference at 4 weeks was 94.7 versus $97.0 \%$ ( $p=0.04$, Fig. $2 \mathrm{e})$. The rates of change of the other variables were not significantly different.

Patient survival in the control and LES groups was 88.3 versus $90.0 \%$, respectively, at 1 year, 56.2 versus $78.2 \%$ at 3 years, and 33.8 versus $26.1 \%$ at 5 years after hepatectomy. Survival rates did not differ significantly between groups ( $p=0.51$, Fig. 3 ). 


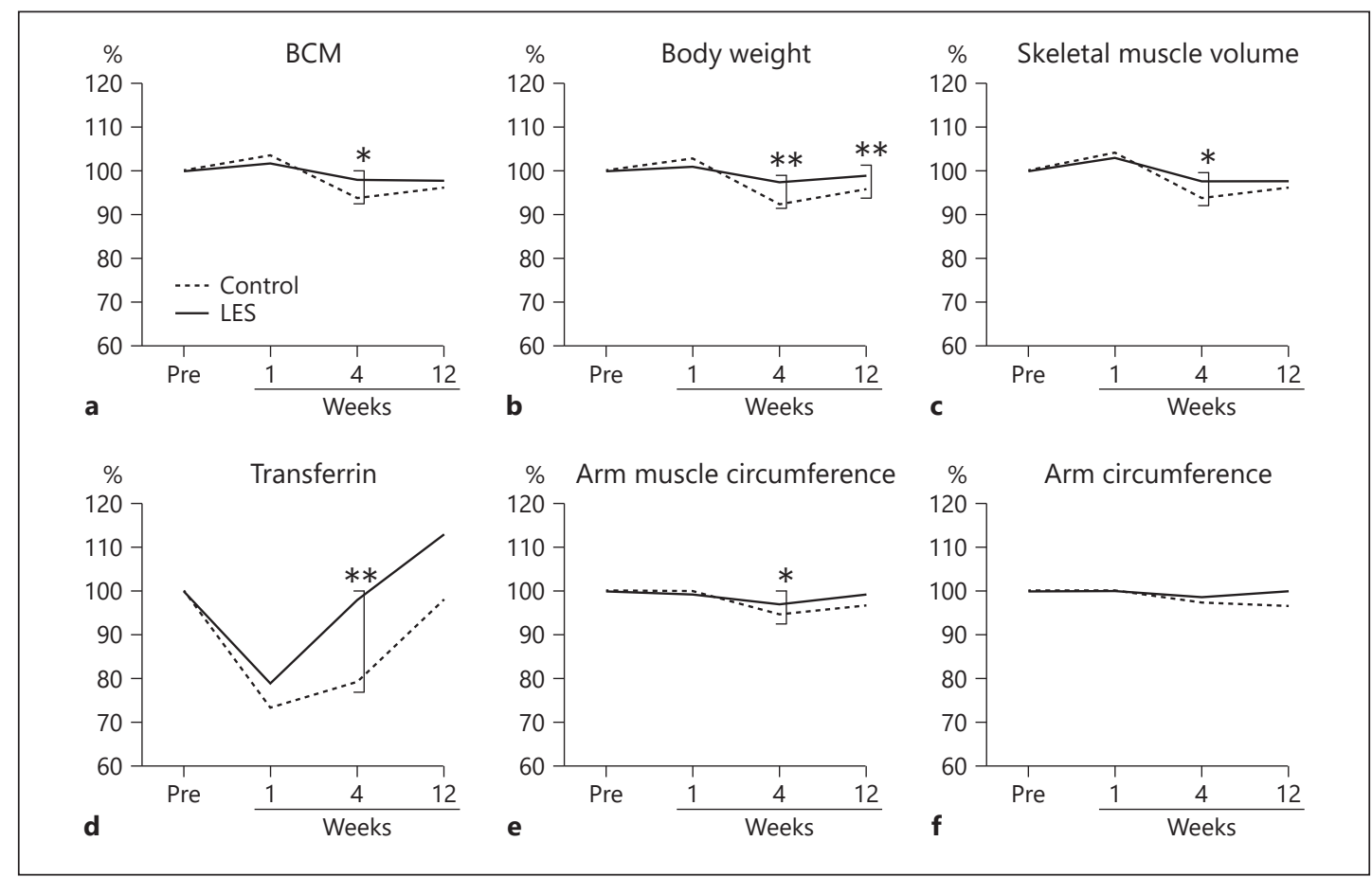

Fig. 2. Change in BCM (a), body weight (b), skeletal muscle volume (c), serum transferrin score (d), arm muscle circumference (e), and arm circumference (f). The change rate of BCM in the control and LES groups was 94.0 versus $98.0 \%$ at 4 weeks $(p=0.03$, a); body weight at 4 and 12 weeks was 93.2 versus $97.6 \%(p<$ 0.01 ) and 96.2 versus $99.1 \%(p<0.01, \mathbf{b})$, respectively; skeletal muscle volume at 4 weeks was 93.9 versus $97.5 \%(p=0.02, \mathbf{c})$; transferrin at 4 weeks was 79.0 versus $97.5 \%(p<0.01, \mathbf{d})$; and arm muscle circumference at 4 weeks was 94.7 versus $97.0 \%(p=0.04, \mathbf{e})$. The change rates of the other variables were not significant. BCM, body cell mass; LES, late-evening snack. * $p<0.05$; ${ }^{*} p<0.01$.

Fig. 3. Overall patient survival. Patient survival did not differ significantly between the control and LES groups at 1,3 , or 5 years after hepatectomy $(p=0.51)$. LES, late-evening snack.

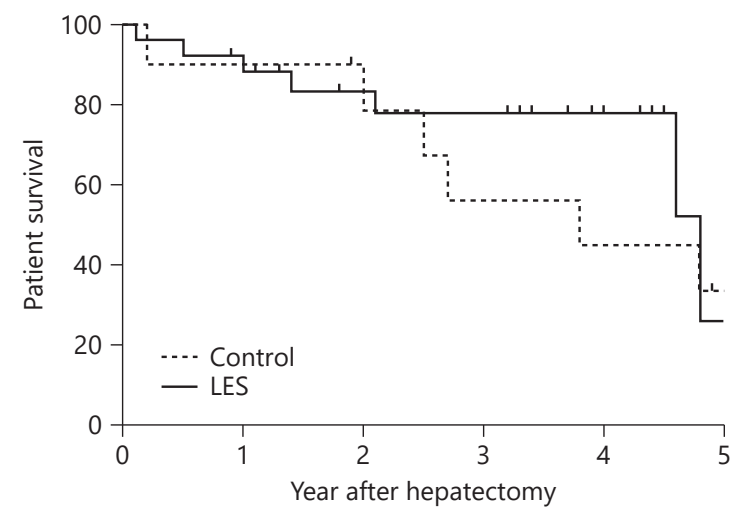

\section{Discussion}

This study is the first to report precise body composition data in relation to the perioperative nutritional status in patients undergoing hepatectomy. Based on body composition analyzed by BIA, supplementation with a 210 -kcal late-evening carbohydrate and BCAA 
snack improved the nutritional status of patients with impaired liver function undergoing hepatectomy in the 4-week period after the operation compared with the control group. The first day of defecation was significantly earlier in the LES group. None of the participants had sarcopenia and severe malnutrition and the study showed no large clinical and data difference between the groups.

The goal of nutritional support is to improve surgical outcome and survival. Few studies, however, have examined the effect of nutritional support for hepatectomy patients both preoperatively and postoperatively. A previous study demonstrated that LES supplementation with BCAA in patients undergoing liver resection for hepatocellular carcinoma led to a shorter hospital stay, higher albumin level, and lower bilirubin level [9]. Perioperative supplementation with a 62 -kcal synbiotic reduced infectious complications in post-hepatectomy patients [10]. A randomized study of patients receiving long-term nutritional support with BCAA reported a decrease in flapping tremor, increased body weight, and improved performance status [11]. Perioperative supplementation with $48 \mathrm{kcal}$ BCAA favorably affected the surgical outcome in liver resection patients by reducing the occurrence of refractory ascites and/or pleural effusion [12]. Another recent randomized, double-blind study, however, showed no clinical advantage of nutritional support for patients undergoing hepatectomy [13]. In the present study, although we found no clinical advantage of supplementation in this small cohort, body composition based on BIA indicated differences in the nutritional status between the groups. The rate of increase in BCM was significantly higher in the LES group than in the control group at 4 weeks after the operation. Further studies with a larger cohort may reveal a clinically meaningful difference.

The administration and/or caloric amount of nutritional support varies by report. In the present study, we selected a 210 -kcal carbohydrate and BCAA snack. In several hepatectomy studies, a 400-kcal snack was selected for supplementation [11, 28, 29]. In a non-surgical setting, Peng et al. [30] reported differences in the amount of daily energy intake and nutritional status of patients with liver cirrhosis. The average daily calorie-based energy intake was 2,198, 2,138, and 1,853 kcal in Child-Pugh A, B, and C patients, respectively. The difference in the intake was $353 \mathrm{kcal}$ between cirrhotic patients with and without significant protein depletion, $285 \mathrm{kcal}$ between Child-Pugh B and C patients, and $345 \mathrm{kcal}$ between Child-Pugh A and C patients. Therefore, supplementation with 200-400 kcal may be appropriate for patients with impaired liver function undergoing hepatectomy.

The body composition of cirrhotic patients indicates protein depletion and accumulation of TBW, even in patients with Child-Pugh class A liver disease [31]. In the present study, all patients were Child-Pugh class A except for 1 patient assigned to the LES group who had a Child-Pugh score 7, class B. The median BCM was relatively low in both groups before the operation. BIA can simultaneously measure several variables of predicted skeletal muscle volume. Although there was no sarcopenia based on a consensus report of the Asian Working Group 2 weeks before the operation, the skeletal muscle volume in the control and LES groups was at the lower limit of the normal range. Body composition based on BIA may precisely disclose potential sarcopenia before the operation. Furthermore, late-evening carbohydrate and BCAA snacks may improve skeletal muscle volume. In the present study, the median skeletal muscle volume 2 weeks before hepatectomy and 4 weeks after the operation was similar in the LES group (from 25.4 to $25.3 \mathrm{~kg}, 0.4 \%$ decrease), but the median skeletal muscle volume in the control group was low before liver resection and had further decreased at 4 weeks after the operation (from 24.0 to $21.9 \mathrm{~kg}, 8.7 \%$ decrease). Although body composition based on BIA may have a high sensitivity to show latent sarcopenia or poor nutritional status, further studies are needed to determine slight differences in the nutritional status in patients with slight-to-moderately impaired liver function who undergo hepatectomy. 
We focused on slight-to-moderately impaired liver function and an ICG retention rate at 15 min greater than 15\% [32] to stratify Child-Pugh class A surgical patients. Limitations of the present study include the tendency toward a greater number of cirrhotic patients in the control group. Although there was no significant difference, only $46 \%$ of patients in the LES group were cirrhotic. The results may have a relation with differences in skeletal muscle volume after surgery. Although there are no similar reports, the study was a non-randomized controlled trial, thus the results should be carefully interpreted. Colorectal liver metastasis patients were different from hepatocellular carcinoma patients in their nutritional backgrounds and etiology of impaired liver function. However, hidden malnutrition of colorectal liver metastasis patients with longer preoperative chemotherapy is still not known and further study is required. The findings of the present study suggest that nutritional support for hepatectomy patients with impaired liver function is promising in this setting and warrants further evaluation. In the present study, $43 \%$ of patients needed an urgent operation without a sufficient preoperative period of nutritional support. In further studies, a suitable sample size should be carefully calculated to ascertain small nutritional differences of not significantly malnourished patients.

In conclusion, late-evening carbohydrate and BCAA snack supplementation may improve the nutritional status of patients with impaired liver function undergoing hepatectomy.

\section{Acknowledgements}

We thank Yoko Uehata for important support with bioelectrical impedance analysis.

\section{Statement of Ethics}

The study was approved by the institutional review board of the University of Tokyo in 2012 (P2012002-11X) and was registered in the University Hospital Information Network clinical trials registry (UMIN000009154; https://center.umin.ac.jp/ctr/index.htm).

\section{Disclosure Statement}

The authors declare no conflicts of interest.

\section{Funding Sources}

This work was supported by grants 17H04279 and 19K09191 from the Ministry of Education, Culture, Sports, Science, and Technology of Japan.

\section{Author Contributions}

K.K. and J.K. designed the study and wrote the initial draft of the manuscript. T.Y., Y.K., J.A., N.A., and T.I. contributed to analysis and interpretation of the data, and assisted in the preparation of the manuscript. K.F., R.S., H.I., and N.Ku. contributed to data collection and interpretation. N.Ko. and K.H. critically reviewed the manuscript. All authors approved the final version of the manuscript. 


\section{References}

1 Kokudo N, Hasegawa K, Akahane M, Igaki H, Izumi N, Ichida T, et al. Evidence-based clinical practice guidelines for hepatocellular carcinoma: The Japan Society of Hepatology 2013 update (3rd JSH-HCC Guidelines). Hepatol Res. 2015;45(2):12464.

2 Karoui M, Penna C, Amin-Hashem M, Mitry E, Benoist S, Franc B, et al. Influence of preoperative chemotherapy on the risk of major hepatectomy for colorectal liver metastases. Ann Surg. 2006 Jan;243(1):1-7.

3 Dokmak S, Ftériche FS, Borscheid R, Cauchy F, Farges O, Belghiti J. 2012 Liver resections in the 21st century: we are far from zero mortality. HPB (Oxford). 2013 Nov;15(11):908-15.

4 Melloul E, Hübner M, Scott M, Snowden C, Prentis J, Dejong CH, et al. Guidelines for Perioperative Care for Liver Surgery: Enhanced Recovery After Surgery (ERAS) Society Recommendations. World J Surg. 2016 Oct; 40(10): 2425-40.

5 Plauth M, Cabré E, Campillo B, Kondrup J, Marchesini G, Schütz T, et al.; ESPEN. ESPEN Guidelines on Parenteral Nutrition: hepatology. Clin Nutr. 2009 Aug;28(4):436-44.

6 Yamauchi M, Takeda K, Sakamoto K, Ohata M, Toda G. Effect of oral branched chain amino acid supplementation in the late evening on the nutritional state of patients with liver cirrhosis. Hepatol Res. 2001;21:199_ 204.

7 Aoyama K, Tsuchiya M, Mori K, Kubo Y, Shiraishi K, Sakaguchi E, et al. Effect of a late evening snack on outpatients with liver cirrhosis. Hepatol Res. 2007;37:608-14.

8 Weimann A, Braga M, Carli F, Higashiguchi T, Hubner M, Klek S, et al. ESPEN guideline: clinical nutrition in surgery. Clin Nutr. 2017;36:623-50.

9 Meng WC, Leung KL, Ho RL, Leung TW, Lau WY. Prospective randomized control study on the effect of branched-chain amino acids in patients with liver resection for hepatocellular carcinoma. Aust N Z J Surg. 1999 Nov;69(11):811-5.

10 Sugawara G, Nagino M, Nishio H, Ebata T, Takagi K, Asahara T, et al. Perioperative synbiotic treatment to prevent postoperative infectious complications in biliary cancer surgery: a randomized controlled trial. Ann Surg. 2006 Nov;244(5):706-14.

11 The San-in Group of Liver Surgery. Long-term oral administration of branched chain amino acids after curative resection of hepatocellular carcinoma: a prospective randomized trial. Br J Surg. 1997 Nov;84(11):1525-31.

12 Kikuchi Y, Hiroshima Y, Matsuo K, Kawaguchi D, Murakami T, Yabushita Y, et al. A Randomized Clinical Trial of Preoperative Administration of Branched-Chain Amino Acids to Prevent Postoperative Ascites in Patients with Liver Resection for Hepatocellular Carcinoma. Ann Surg Oncol. 2016 Oct;23(11):3727-35.

13 Seguin P, Locher C, Boudjema K, Hamon C, Mouchel C, Malledant Y, et al. Effect of a Perioperative Nutritional Supplementation with Oral Impact ${ }^{\circledR}$ in Patients undergoing Hepatic Surgery for Liver Cancer: A Prospective, Placebo-Controlled, Randomized, Double-Blind Study. Nutr Cancer. 2016;68(3):464-72.

14 Crawford DH, Shepherd RW, Halliday JW, Cooksley GW, Golding SD, Cheng WS, et al. Body composition in nonalcoholic cirrhosis: the effect of disease etiology and severity on nutritional compartments. Gastroenterology. 1994 Jun;106(6):1611-7.

15 Lee YH, Lee JD, Kang DR, Hong J, Lee JM. Bioelectrical impedance analysis values as markers to predict severity in critically ill patients. J Crit Care. 2017 Aug;40:103-7.

16 Pirlich M, Schütz T, Spachos T, Ertl S, Weiss ML, Lochs H, et al. Bioelectrical impedance analysis is a useful bedside technique to assess malnutrition in cirrhotic patients with and without ascites. Hepatology. 2000 Dec; 32(6):1208-15.

17 Kawaguchi T, Taniguchi E, Itou M, Ibi R, Okada T, Mutou M, et al. Body cell mass is a useful parameter for assessing malnutrition and severity of disease in non-ascitic cirrhotic patients with hepatocellular carcinoma or esophageal varices. Int J Mol Med. 2008 Nov;22(5):589-94.

18 Plauth M, Cabré E, Riggio O, Assis-Camilo M, Pirlich M, Kondrup J, et al.; DGEM (German Society for Nutritional Medicine); ESPEN (European Society for Parenteral and Enteral Nutrition). ESPEN Guidelines on Enteral Nutrition: liver disease. Clin Nutr. 2006 Apr;25(2):285-94.

19 Yamaguchi T, Kokudo T, Akamatsu N, Kaneko J, Arita J, Sakamoto Y, et al. Liver Regeneration is Preserved After At Least Four Repeated Liver Resections for Hepatocellular Carcinoma. World J Surg. 2018 Dec;42(12):407080.

20 Shirata C, Hasegawa K, Kokudo T, Arita J, Akamatsu N, Kaneko J, et al. Surgical site infection after hepatectomy for hepatocellular carcinoma. Dig Surg. 2018;35(3):204-11.

21 Tani K, Shindoh J, Akamatsu N, Arita J, Kaneko J, Sakamoto Y, et al. Management of disappearing lesions after chemotherapy for colorectal liver metastases: relation between detectability and residual tumors. J Surg Oncol. 2018;117(2):191-7.

22 Imamura H, Seyama Y, Kokudo N, Maema A, Sugawara Y, Sano K, et al. One thousand fifty-six hepatectomies without mortality in 8 years. Arch Surg. 2003 Nov;138(11):1198-206.

23 Mullen JT, Ribero D, Reddy SK, Donadon M, Zorzi D, Gautam S, et al. Hepatic insufficiency and mortality in 1,059 noncirrhotic patients undergoing major hepatectomy. J Am Coll Surg. 2007 May;204(5):854-62.

24 Mangram AJ, Horan TC, Pearson ML, Silver LC, Jarvis WR: Guideline for Prevention of Surgical Site Infection, 1999. Centers for Disease Control and Prevention (CDC) Hospital Infection Control Practices Advisory Committee. Am J Infect Control. 1999;27:97-134. 
25 Chen LK, Liu LK, Woo J, Assantachai P, Auyeung TW, Bahyah KS, et al. Sarcopenia in Asia: consensus report of the Asian Working Group for Sarcopenia. J Am Med Dir Assoc. 2014 Feb;15(2):95-101.

26 Baker JP, Detsky AS, Wesson DE, Wolman SL, Stewart S, Whitewell J, et al. Nutritional assessment: a comparison of clinical judgement and objective measurements. N Engl J Med. 1982 Apr;306(16):969-72.

27 Dindo D, Demartines N, Clavien PA. Classification of surgical complications: a new proposal with evaluation in a cohort of 6336 patients and results of a survey. Ann Surg. 2004 Aug;240(2):205-13.

28 Okabayashi T, Nishimori I, Yamashita K, Sugimoto T, Namikawa T, Maeda H, et al. Preoperative oral supplementation with carbohydrate and branched-chain amino acid-enriched nutrient improves insulin resistance in patients undergoing a hepatectomy: a randomized clinical trial using an artificial pancreas. Amino Acids. 2010 Mar;38(3):901-7.

29 Ishikawa Y, Yoshida H, Mamada Y, Taniai N, Matsumoto S, Bando K, et al. Prospective randomized controlled study of short-term perioperative oral nutrition with branched chain amino acids in patients undergoing liver surgery. Hepatogastroenterology. 2010 May-Jun;57(99-100):583-90.

30 Peng S, Plank LD, McCall JL, Gillanders LK, Mcllroy K, Gane EJ. Body composition, muscle function, and energy expenditure in patients with liver cirrhosis: a comprehensive study. Am J Clin Nutr. 2007 May;85(5):1257-66.

31 Prijatmoko D, Strauss BJ, Lambert JR, Sievert W, Stroud DB, Wahlqvist ML, et al. Early detection of protein depletion in alcoholic cirrhosis: role of body composition analysis. Gastroenterology. 1993 Dec;105(6):183945.

32 Kusaka K, Harihara Y, Torzilli G, Kubota K, Takayama T, Makuuchi M, et al. Objective evaluation of liver consistency to estimate hepatic fibrosis and functional reserve for hepatectomy. J Am Coll Surg. 2000 Jul;191(1): 47-53. 